\title{
ANALYSIS OF A COHORT USING JAK INHIBITORS FOR RHEUMATOID ARTHRITIS: AN ONGOING OBSERVATIONAL PROSPECTIVE STUDY
}

Lucas Moyses Carvalho de Oliveira1, , Maria Fernanda Brandão de Resende ${ }^{1}$, Maria Raquel da Costa Pinto ${ }^{1}$, Débora Cerqueira Calderaro ${ }^{1}$, Adriana Maria Kakehasi ${ }^{1}$, João Victor de Pinho Costa ${ }^{1}$, Clarice Moura Mata Machado ${ }^{1}$, Rafael Fusaro Aguiar Oliveira ${ }^{1}$

1.Universidade Federal de Minas Gerais, Belo Horizonte (MG), Brazil.

*Corresponding author: moyses_lucas@hotmail.com

\section{BACKGROUND}

Janus kinase inhibitors (JAKi) are indicated as second-line therapy for rheumatoid arthritis (RA), after synthetic or biologic failure, with proven efficacy and safety in clinical trials. However, evaluation of JAKi in local RA populations is necessary, through real life studies.

\section{METHODS}

An analysis of 35 RA patients with history of JAKi therapy in the last 5 years was made, enrolled in an ongoing observational cohort, with appointments at baseline and follow-up at 3, 6, and 12 months.

\section{RESULTS}

Thirty (86\%) patients were women, 24 (68.5\%) had high rheumatoid factor and/or anti-CCP titers, 4 (11.5\%) presented extraarticular disease (ocular or pulmonary), 7 (20\%) had rheumatoid nodules, and 22 (63\%) had radiographic erosions at the beginning of JAKi. Mean age was 52.77 ( \pm 11.54$)$ years and RA duration $15.26( \pm 9.21)$ years. Total time using JAKi was 14.5 months (1-60), and the median number of treatment schemes before JAKi was 4 (2-8). There was a significant reduction on DAS28 and CDAI at 6 months compared to baseline, but not at 3 months (Table 1). Prednisone median dose was lower at 12 months compared to baseline (5 [0-20] vs. 5 [0-10] mg/day, $p=0.044)$. Ten patients $(28.5 \%)$ discontinued treatment due to secondary failure $(n=6)$ or adverse events $(n=4)$, such as myalgia $(n=1)$, thrombosis $(n=2)$ and zoster $(n=2)$. Other adverse events were nausea $(n=2)$, repeated furunculosis $(n=1)$ and transitory transaminase elevation $(n=1)$. Patients with longer disease duration tended to be more likely to discontinue JAKi (19.4 [ \pm 10.69$]$ vs. $13.6[ \pm 8.21]$ years, $p=0.09$ ).

Table 1. Baseline mean (and standard deviation) DAS28 and CDAI compared to 3-, 6- and 12-month follow-up.

\begin{tabular}{ccc}
\hline & DAS28 & CDAI \\
\hline Baseline vs. 3 months & $4.49( \pm 1.2)$ vs. 3.83 $( \pm 1.25), p=0.06(n=27)$ & $4.49( \pm 1.2)$ vs. 3.83 $( \pm 1.25), p=0.06(n=21)$ \\
\hline Baseline vs.6 months & $4.38( \pm 1.41)$ vs. 3.34 $( \pm 1.075), p=0.007(n=22)$ & $4.37( \pm 1.4)$ vs. 3.34 $( \pm 1.07), p=0.007(n=19)$ \\
\hline Baseline vs. 12 months & $4.67( \pm 1.46)$ vs. 3.91 $( \pm 1.11), p=0.036(n=17)$ & $4.67( \pm 1.46)$ vs. 3.91 $( \pm 1.11) p=0.036(n=16)$ \\
\hline
\end{tabular}

\section{CONCLUSION}

In this study, JAKi effectively reduced disease activity scores and allowed corticosteroids tapering up to 12 months of therapy, with an acceptable safety profile, despite relatively high dropout rate. It is reasonable to wait for 6 months to evaluate efficacy in this multifailed group. Limitations include missing data at 6 - and 12-month appointments, short follow-up period, and small sample. The progression of study recruitment will allow further analysis and conclusions.

\section{KEYWORDS}

JAK inhibitors, Rheumatoid arthritis, Cohort. 